\section{Parenting stress and impact of illness in parents of children with coeliac disease}

\author{
Maria Stella Epifanio, Vitalba Genna, \\ Maria Grazia Vitello, Michele Roccella, \\ Sabina La Grutta \\ Departments of Psychology, University \\ of Palermo, Italy
}

\begin{abstract}
Coeliac disease (CD) is a chronic disease which could stress patients and their family. Although, poor attention has been paid to the quality of life in CD children and to the functioning of families with CD children. The study aims to evaluate the parenting perception of the CD impact and the parenting distress level. A group of 74 parents of CD children compiled the Impact Childhood Illness Scale and the Parenting Stress Index which is also compiled by 74 parents of health children. The assessment does not reveal a significant impact of CD on patient's personal life although some critical areas emerged. Results evidenced an higher level of parenting stress in parents of CD children than parents of healthy children. $\mathrm{CD}$, if suitably managed, has not a critical impact on parenting perception. Although, CD certainly put parents through an higher risk of a distress related to parenting role than parents with health children. A early identification of parenting distress in a pediatric chronic illness could facilitate the adjustment to pathology.
\end{abstract}

\section{Introduction}

Coeliac disease (CD) is a widespread immune-mediated systemic disorder with genetic basis. It compromises the functioning of small intestine which incurs in a chronic inflammation with the consequent disappearance of intestinal villi, after taking food with gluten. ${ }^{1}$ It manifests in genetically susceptible individuals and it is elicited by ingestion of gluten, a protein present in cereals like wheat, oats, barley and rye. Although the significant increase of its incidence in the $20^{\text {th }}$ century, the diagnosis of coeliac disease (CD) is underestimated: it is estimated that each 7 individuals 1 remains not diagnosed.2 CD occurs in general European population with a prevalence of $1 \%$ and in Italy with a prevalence of $0.7 \%{ }^{2}$

The reasons of this underestimation depend on patient ability to suspect the disease, on the availability of diagnostic infrastructures and on the variability in clinical intensity.

Diagnosis could be delayed or never made due a clinical manifestation different from the classic one or due a lack of symptoms. ${ }^{3}$ Indeed, clinical range is extremely heterogeneous and symptomatic signs change both in intensity/ gravity.

Most frequent symptoms are chronic abdominal pain, swollen abdomen, chronic or intermittent diarrhea, weight and strength loss, iron-deficiency anemia, nausea or vomiting, cramping or distension, stunted growth, failure to thrive, delayed puberty, chronic constipation, chronic fatigue, recurrent aphthous, dermatitis herpetiformis (symptomatic form). When the illness is asymptomatic, the risk conditions for CD are type 1 diabetes mellitus, Down syndrome, autoimmune thyroid disease, Turner syndrome, Williams syndrome, selective immunoglobulin A deficiency, autoimmune liver disease, and first-degree relatives with CD.4-7

The only effective therapy is a strictly gluten-free diet which has prodigious and immediate effects on coeliac patients: the disappearance of clinical manifestations, the normalization of blood tests, the structural restoration of intestinal mucosa and the fast improvement of appetite and mood. The possible metabolic alterations (reduced bone mineralization, iron deficiency) slowly return to normal levels. An early dietary treatment minimizes also the risk of long term complications, but it doesn't delete the possibility of complications derived from other disease in comorbidity. ${ }^{6}$ Since the symptomatic and therapeutic features above described, the CD involves the whole bio-psycho-social functioning of the subject.

The cultural, emotional and psychological importance of food and feeding makes the CD as a pathology with a strong psychological impact on the patient. The food involves rites and traditions, festivities and religions, and it is a vehicle of conscious and unconscious meanings able to influence the whole lifestyle of a subject. Feeding represents the first social recurrent activity and the first type of dialogue mother-child and an essential aspect in the children's care, not only for the physical growth, but also for the affective feeding and exchange which contribute to the creation of a secure attachment bond. ${ }^{8}$ During feeding, the child is in the better conditions - also the physical ones, relating to the distance from the significant other - to meet a wide range of affective stimuli. Through the feeding relation between baby/infant and caregiver, psychological bases of identity and personality develop. ${ }^{9}$

Considering the importance of feeding activity, an good feeding interaction represents a source of satisfaction and gratification while possible troubles in this area cause an
Correspondence: Maria Stella Epifanio, Department of Psychology, University of Palermo, via delle Scienze, 90128 Palermo, Italy.

Tel. +39.091.238.97714 - Fax. +39.091.651.3825.

E-mail: mariastella.epifanio@unipa.it

Key words: coeliac disease, parenting stress, chronic disease, impact childhood illness.

Acknowledgments: we are grateful to all parents, pediatricians and chemists who took part in the research project.

Contributions: the authors contributed equally.

Conflict of interests: the authors declare no potential conflict of interests.

Received for publication: 3 October 2013. Accepted for publication: 4 November 2013.

This work is licensed under a Creative Commons Attribution NonCommercial 3.0 License (CC BYNC 3.0).

(C) Copyright M.S.Epifanio et al., 2013

Licensee PAGEPress, Italy

Pediatric Reports 2013; 5:e19

doi:10.4081/pr.2013.e19

intensive worry. ${ }^{9}$ Being forced to modify own diet means also to have to modify own relational and social habits and it influences the nature of affective ties and the development sense of somatic experience, the basis of identity development, especially in childhood.

Diet derives from the Greek term diaita which means way, style, standard of life and a gluten-free diet refers exactly to the change of the lifestyle not only of the coeliac subject, but also of his life environment. ${ }^{10}$ For this reason, the CD diagnosis requires a change from a condition of illness to a condition of life.

International researches about $\mathrm{CD}$ have mainly concentrated on medical aspects with poor attention to psychological ones. The psychological attention almost exclusively concentrated on adult with $\mathrm{CD}, 11$ on their emotional reactions, on their defense mechanisms and on their attitude to the imposed diet, 12 but poor attention has been paid to the quality of life in $\mathrm{CD}$ children and to the functioning of families with CD children/adolescents. ${ }^{13} \mathrm{~A}$ possible explanation of it is the lack of standardized tools for their assessment, especially in Italian validation.

When the patient is a child, the parents take the emotional weight of the diagnosis and the food therapy, ${ }^{14}$ and the parenting support is an important factor for patient's health condition. Indeed, the family support has a substantial role in the treatment adherence of a child with a chronic disease and we think this is more evident in the CD,15 in which the daily therapy 
concerns only the diet. A study have evidenced that about $20 \%$ of the coeliac patients' parents state that the whole family, often or always, eats gluten-free. ${ }^{16}$ When a chronic illness influences the feeding and requires the continuous parenting attention, parents can feel not able to feed suitably their child. The necessary reorganization of the activities and family habits could be a source of stress for the CD child's family, such as in other chronic illness. ${ }^{17-19}$ The parenting stressful condition concerns both the received diagnosis and the prescribed therapy; parents admit the important responsibility, but not always they seem or feel prepared. ${ }^{20}$ Indeed, having to pay attention to the food contamination is experienced by many parents as a stressful condition and it is often faced with insecure, anxious and overcontrolling care attitudes, related to feeding and not only. ${ }^{16}$

Basing on these considerations, the study aims to evaluate, in a group of CD children's parents: i) the parenting perception of the impact of the chronic pediatric disease; ii) the level of parenting distress; iii) the relationship between parenting distress and parenting perception of the impact of the chronic pediatric disease. The study also aims to verify iv) if there are significant differences, in terms of parenting distress, between parents with CD children and parents with health children.

Basing on scientific literature and clinical experience in chronic diseases, ${ }^{18-23}$ we hypothesize that, parents with CD children feel an increased parenting distress and an increased preoccupation about the impact of chronic illness on present and future live of the children.

\section{Materials and Methods}

A group of 74 parents (28 fathers and 46 mothers, $M=37.7$ years) of children (2-12 years old) with diagnosis of CD were selected in some pediatric surgeries in Agrigento and in a pharmacy well-known due to the wide availability of gluten free products.

A group of 74 parents (22 fathers and 52 mothers, $M=35.8$ years) of health children (212 years old) were recruited with snowball technique.

When the research aims were explained and the informed consent was collected, parents of the clinical group compiled a socio-demographic questionnaire and two self-reports: the Impact Childhood Illness Scale (ICIS) and the Parenting Stress Index-SF (PSI-SF).24-26 The control group compiled only the socio-demographic questionnaire and PSI-SF.

The ICIS is a self-administered questionnaire used to assess the impact of infantile illness on parents. It was created to investigate the feelings of epileptic children's parents, ${ }^{24}$ but the authors suggest its use also with other chronic illnesses, because none of the items specifically mentions this pathology. Each of the 30 items investigates at the same time two dimensions: the frequency of a problem and the degree of the parenting concerns that it causes. For both dimensions a Likert scaling method (never, sometimes, often) is used. The items are divided in 4 sections, which respectively investigate: i) the impact of the illness and its treatment; ii) the impact on the growth and the child's adaptation; iii) the impact on the parents; iv) the impact on the family.

The total score (range 0-60) is the sum of the four sections scores and of the two dimensions (30 for each one). High scores indicate an high frequency of problems perceived by parents and of an high concerns that they cause. The PSI-SF is the short version of the Parenting Stress Index $2,{ }^{25,26}$ a greatly used test to value the parenting stress, defined by the authors as the constellation of bio-psychosocial factors (anxiety, uncomfortable, psychological and emotional tension, negative/maladaptive coping to stressful events, emotional incompetence, etc.) which distort the normal physiological reaction to a stressor and predispose the subject to high psychological, sociorelational, etc. vulnerability. ${ }^{26}$ In the test, the parent expresses the degree of agreement/disagreement with the 36 statements (answer in a Likert scale of 5 points).

The test uses three factors to measure parenting stress, to which correspond the three subscales: i) the scale of parenting Distress (PD): it defines the level of distress which a parent lives in his parenting role, understood as derived from personal factors directly linked to this role; ii) the scale of the dysfunctional Interaction parent-child (P-CDI): it values the parenting perception of a child not responding to his expectations and of an interaction with the child neither reinforcing nor rewarding; iii) the scale of the Difficult Child (DC): it values how much the parent perceives his child as easy/difficult to manage, basing on some of his behavioral characteristics.

The total score, obtained by the sum of the scores at the 3 subscales, can be interpreted as

Table 1. Descriptive analysis about ICIS scores reported by parents with coeliac disease children $(\mathrm{N}=62)$.

\begin{tabular}{lcc} 
ICIS subscales & $\begin{array}{c}\text { Frequency } \\
\text { dimension } \\
\text { M (SD) }\end{array}$ & $\begin{array}{c}\text { Concerns } \\
\text { dimension } \\
\text { M (SD) }\end{array}$ \\
Impact of the illness and its treatment & $0.68(0.5)$ & $0.84(0.7)$ \\
Impact on the growth and the child's adaptation & $2.77(1.5)$ & $3.00(2.2)$ \\
\hline Impact on the parents & $2.74(1.3)$ & $2.87(1.8)$ \\
Impact on the family & $4.68(2.7)$ & $3.90(3.8)$ \\
\hline Total & $10.87(4.6)$ & $10.61(7.5)$ \\
\hline
\end{tabular}

a stress index related to the only parenting role, in its three sub-dimensions (an higher score indicates a greater perceived stress). nificant stress, both for the global scale and for the three subscales, is $85 \%$.

The test includes also a Defensive Responding scale, useful to control the validity of the protocol, which indicates if the parent tends to give a better self-image, playing down the problems and the perceived stress in the relationship with the child (score $\leq 10$ ).

When the data were collected, we carried out the statistical descriptive on ICIS and PSI-SF scores (aim 1 and aim 2).

Concerning the parenting perception of the CD impact, with correlation analyses, we analyzed the relationship between the parenting answers to the frequency dimension and to concerns one, in order to demonstrate the consistency between the two dimensions. To a better understanding of the parenting perception of illness impact we conducted a detailed analysis of ICIS items checking the answers frequency at every items. Concerning parenting distress, we preliminarily evaluated protocols validity analysis through Defensive Responding scale, we rejected invalid protocols, and we carried out the descriptive analyses on the scores of remaining protocols. Furthermore, to compare clinical and control group results (aim 3), we carried out statistical analysis with Chi Square test. Every analyses were carried out using the Statistical Package for Social Science software.

\section{Results}

On the base of Defensive Responding scale scores, 12 protocols of CD group and 14 of control group were excluded from statistical analysis.

Concerning the evaluation of the impact of the pediatric illness in clinical group (aim 1), average scores both in the frequency dimension and in the concerns one are low (Table 1). The critical cut-off to indicate a clinically sig- 
Interesting information about the impact of illness emerged during the analysis of ICIS subscales scores (Table 1): the lowest scores, both in frequency dimension and in concerns dimension, emerged in the first subscale the impact of the illness and its treatment while the highest ones were reported in the last subscale, relating to the impact of the illness on the whole family (Table 1). Correlations between the two ICIS dimensions scores, calculated with Spearman's rho at each subscale, are significant in every subscales and it varies from $r=0.462$ to $r=0.705 \mathrm{P}<0.01$ ) (Table 2).

The detailed analysis of ICIS items suggests that parents of CD children reported higher frequency of often answers in some items (like item 2, 6, 8, 20 and 29) than other ones (Supplementary Table 1). Concerning parent- ing distress (aim 2), 28 parents with CD children (versus 16 in the control group) obtained a score equal or higher than the $85 \%$ in the PSI-SF global score and the most critical scores are reported by clinical group in Difficult Child subscale (Table 3). Regarding aim 3, total PSISF score correlated with ICIS total concerns dimension score $(\mathrm{r}=3.73 ; \mathrm{P}<0.01)$, with concerns dimension the impact on the parents $(\mathrm{r}=3.98 ; \mathrm{P}<0.01)$, with concerns dimension the impact on the family $(\mathrm{r}=3.47 ; \mathrm{P}<0.01)$. Every PSI-SF subscale scores correlated with some ICIS subscale scores (Table 2). At Chi square test, significant differences between the two groups emerged in Total PSI-SF $\left(\chi^{2}(36)=68.6\right.$; $\mathrm{P}=0.01)$ in PD subscale $\left(\chi^{2}(22)=51.6 ; \mathrm{P}=0.000\right)$ in P-CDI one $\left(\chi^{2}{ }_{(18)}=39.7 ; \mathrm{P}=0.02\right)$ and in DC one $\left(\chi^{2}{ }_{(24)}=41.4 ; \mathrm{P}=0.015\right)$.

\section{Discussion and Conclusions}

The presence of invalid protocols indicates parenting defensiveness and suggests that the parent tends to give a better self-image, playing down the problems and the perceived stress in the relationship with the child.

Concerning parenting perceptions about the illness impact, no specific critical areas emerged.

The low results relating to the first subscale suggest that CD treatment, because they are not pharmacological, does not represent a problematic aspect in the management of the CD such as measured by ICIS. Its items do not suitably value the anxiety and the worry generally connected to this pathology and they do not

Table 2. Correlations between the two ICIS subscales dimensions, and between PSI-SF and ICIS scores reported by parents with CD children (N=64).

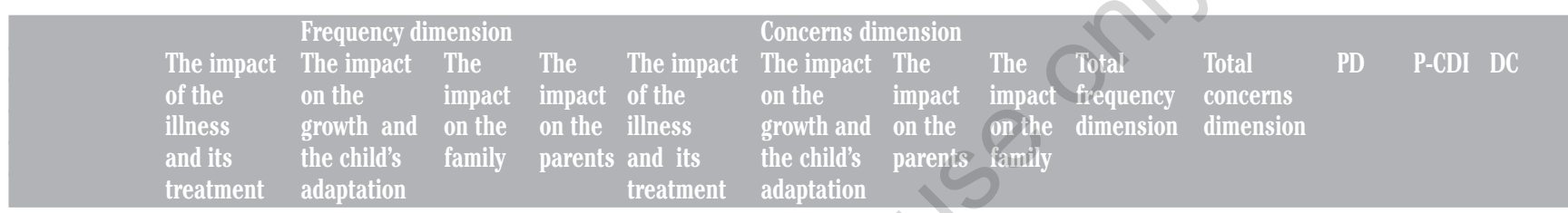

The impact $\quad 1$

of the illness

and its

treatment

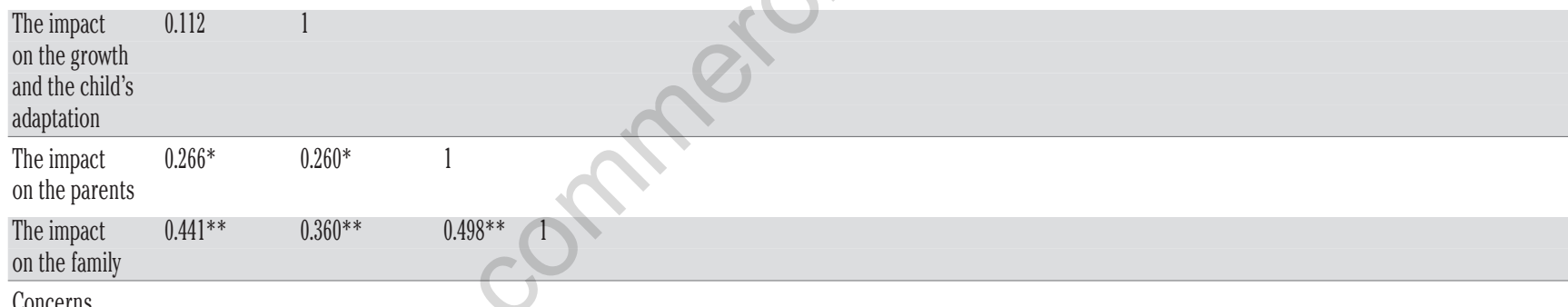

dimension

\begin{tabular}{l}
$\begin{array}{l}\text { The impact of } \\
\text { the illness } \\
\text { and its } \\
\text { treatment }\end{array}$ \\
\hline $\begin{array}{lllllll}\text { The impact } & -0.462^{* *} & 0.266^{*} & 0.039 & 0.158 & 1 & \\
\end{array}$
\end{tabular}

on the growth

and the child's

adaptation

\begin{tabular}{|c|c|c|c|c|c|c|c|c|c|c|c|}
\hline $\begin{array}{l}\text { The impact } \\
\text { on the parents }\end{array}$ & 0.055 & 0.234 & $0.705^{* *}$ & $0.356^{* *}$ & 0.167 & $0.691^{* *}$ & 1 & & & & \\
\hline $\begin{array}{l}\text { The impact } \\
\text { on the family }\end{array}$ & -0.033 & 0.194 & $0.354^{* *}$ & $0.469 * *$ & $0.299 *$ & $0.774^{* *}$ & $0.698 * *$ & 1 & & & \\
\hline $\begin{array}{l}\text { Total frequency } \\
\text { dimension }\end{array}$ & $0.492^{* *}$ & $0.655^{* *}$ & $0.710^{* *}$ & $0.882^{\text {** }}$ & 0.255 & $0.489 * *$ & $0.504^{* *}$ & $0.429 * *$ & 1 & & \\
\hline $\begin{array}{l}\text { Total concerns } \\
\text { dimension }\end{array}$ & 0.040 & $0.347^{* *}$ & $0.479 * *$ & $0.441^{* *}$ & $0.421^{* *}$ & $0.908 * *$ & $0.828 * *$ & $0.941^{* *}$ & $0.518 * *$ & 1 & \\
\hline PD & $0.418^{* *}$ & -0.150 & 0.195 & $0.312^{*}$ & 0.194 & 0.116 & $0.300^{*}$ & $0.322 *$ & 0.229 & $0.292 *$ & 1 \\
\hline P-CDI & -0.002 & $0.275^{*}$ & $0.267^{*}$ & 0.130 & 0.210 & $0.288^{*}$ & $0.428 * *$ & $0.267^{*}$ & 0.254 & $0.352^{* *}$ & $0.351^{* *} 1$ \\
\hline DC & $0.334 *$ & 0.128 & 0.186 & 0.226 & $0.341^{* *}$ & 0.179 & $0.306^{*}$ & $0.279 *$ & $0.270^{*}$ & $0.308 *$ & $0.679 * * 0.530 * * 1$ \\
\hline TOT. PSI & $0.335 *$ & 0.076 & 0.249 & $0.278 *$ & $0.304 *$ & 0.219 & $0.398 * *$ & $0.347^{* *}$ & $0.299 *$ & $0.373^{* *}$ & $0.852 * * 0.694 * * 0.916 * *$ \\
\hline
\end{tabular}


seem to be good indexes of measurement of the impact.

The detailed analysis of ICIS items (Supplementary Table 1) suggests that the parenting preoccupations regards the risk he may injure himself (item 2) and it could regard the fear of the food contamination. In the second subscale the answers at: My child is more moody because of his illness (item 6) and Because of my child's illness, he is teased and bullied (item 8) evidence that parents are preoccupied that the illness has an impact on the child's internal affective world and his sensitivity. The most frequent concern regards the explanation of the illness to the child himself (as measured by item 20) and it could be associated with parenting difficulties to find an explanation, emotionally satisfactory, of the causes of the illness and to accept profoundly its incurability. Moreover, the young age of children can make difficult for the parents the explanation of why I am ill. The younger children are probably not yet able to understand cognitively the meaning of their food restrictions and they could tend to want to try new foods. It can be difficult for a parent to explain why you can't!, which sometimes can take a punitive value. With the growth, children become more cognitively able to understand the meaning of being ill and the meaning of the food restrictions. However, they can became more conscious of the difference compared with peers, especially with the admission to school and in adolescence. Indeed, the adolescent prepares himself to cope with the hard body and identity transformation, process in which he has to include the sense and the meaning to give to his chronic illness.

The scores of the last subscale suggest that for parents the illness has a greater impact on the whole family than that it has respectively on the child or the parents. This evidence suggests that chronic illness is an event requiring a family reorganization in order to respond to the internal and external changes due to the pathology and to the care needs, as measured by item 29 (Supplementary Table 1). The management of the infantile chronic illness is a very important question for parents, not only in the terms of continuity of the effort required, but also in the terms of worry, anxiety and stress, for this special parenting task.

The family experiences some difficulties in physical and affective caregiving but the parents not renounce to the extra-familial activities which indicate a good adaptation in the social context.

Concerning parenting perceptions about the illness impact, we finally believe that the test is not generally sensitive to assess CD impact because this illness does not probably impact on friendly relationships, extra-curricular interests, academic performance, worry concerning the career, physical impairment and

Table 3. Descriptive analysis about PSI-SF and its subscales scores reported by parents with celiac disease children $(\mathrm{N}=62)$ and parents of control group $(\mathrm{N}=60)$

\begin{tabular}{|c|c|c|c|c|}
\hline & \multicolumn{2}{|c|}{ Coeliac disease } & \multicolumn{2}{|c|}{ Control group } \\
\hline & $<85^{\circ}$ perc. & $>85^{\circ}$ perc. & $<85^{\circ}$ perc. & $>85^{\circ}$ perc. \\
\hline Total Parenting stress & 34 & 28 & 44 & 16 \\
\hline Difficult Child (DC) & 20 & 42 & 34 & 26 \\
\hline Parenting Distress (PD) & 46 & 16 & 48 & 12 \\
\hline Interaction parent-child (P-CDI) & 54 & 8 & 50 & 10 \\
\hline
\end{tabular}

love life that ICIS evaluates.

Nevertheless, the test gave us information about some critical points towards which we can direct next researches such as the study of other areas related to the child development of affect and identity.

Concerning parenting distress, the results about the total stress indicates that around half of the parents experience a critical level of parenting stress that is significantly different between the two groups. The frequencies of PD and P-CDI scores higher than $85 \%$ indicate that the sense of parenting responsibility, the relation with the partner, the social support, the mood condition, parent-child dysfunctional interactions, and the perception of themselves as poor sensitivity to children needs are not much altered in CD children's parents. Although, scores at these subscales were significantly higher than control group scores.

The high score at DC scale highlights how the parents refer a lot of difficulties in their CD child's management both putting restrictions to the child and trying to have his collaboration.25,26 This suggests that parents of clinical group, differently of control group, perceive their children as very difficult to manage because they perceive their children's behaviors (temperamental and/or acquired one) characterized by defiance, disobedience and excessive requests. Common requests could concern the specific attentions in food preparation and the continuous supervision on the feeding.

The correlation between parenting stress and the concerns dimension about the CD impact on the family and on the parents suggests that the parents experience difficulties in the carrying out of the parenting tasks and in the family management. Results evidence that parenting concern does not regard the CD influence on the future child development but the most principal parenting stress source regards the parent-child interaction. Indeed, the parents perceive their child as not responding to their expectations and this interaction does not neither reinforce nor reward them.

In conclusion, in one hand the low ICIS scores demonstrate that the test is not sensitivity to assess quality of life in CD children, in the other hand PSI-SF scores confirm that clinical group's parents bring into question their parenting competence and that the most critical dimension regards children characteristics. It is possible that this evidence could be related with the presence of CD and its treatments which could hinder parenting tasks.

Finally, the parenting perception about the children suggests both that the illness represents a possible source of parenting stress and that the illness could influence the child's psychological acquired characteristics.

Highlighting the partiality of an exclusively medical model, the study suggests the need of a psychological expertise in the care of $\mathrm{CD}$ child. It means to consider the patient complexity, in terms of maladaptive and resilient aspects, and the family context.

Indeed, the study focused on feelings of parents with CD children and on their relationship with child wellbeing, which are not yet much explored in literature. A possible mediator variable between parenting stress and child wellbeing could be the adherence to therapeutic regimen (the diet in the $\mathrm{CD}$ ) which has a strong influence on the growth and the health condition of the CD child. When patient is a child, the parenting support plays an important role in treatments adherence, and an optimal disease management requires the parenting consciousness of the own new role and of the change from the condition of parent to that of caregiver, that is of specific care provider.

Finally, we also believe that the evaluation of the impact of the illness and of the parenting stress can be considered clinically significant indexes of the Quality of life (QoL) of the CD child and his family, which are not yet much explored in literature. A preventive identification of these factors could represent another important applicative implication of this study.

\section{References}

1. Fasano A, Catassi C. Current approaches to diagnosis and treatment of celiac disease: an evolving spectrum. Gastroenterology 2001;120:636-51.

2. Mustalahti K, Catassi C, Reunanen A, et al. Coeliac EU Cluster, Project Epidemiology. The prevalence of celiac disease in Europe: results of a centralized, interna- 
tional mass screening project. Ann Med 2010;42:587-95.

3. Bottaro G, Cataldo F, Rotolo N, et al. The clinical pattern of subclinical/silent celiac disease: an analysis of 1026 consecutive cases. Am J Gastroenterol 1999;94:691-6.

4. Hadjivassilou M, Gibson A, Davies-Jones $\mathrm{GA}$, et al. Associated with the cultivation of wheat. J Pediatr Gastroenterol Nutr 1997;24:S14-6.

5. Accomando S, Albino C. La malattia celiaca: nuove implicazioni in una antica condizione. Celiac disease: new implications in an old condition. Capsula Eburnea 2006;2:1-5.

6. Husby S, Koletzko S, Korponay-Szabò IR, et al. European Society for Pediatric Gastroenterology, Hepatology, and Nutrition guidelines for the diagnosis of coeliac disease. J Pediatr Gastroenterol Nutr 2012;54:136-60.

7. Garampazzi A, Rapa A, Mura S, et al. The clinical pattern of celiac disease is still changing? J Pediatr Gastroenterol Nutr 2007;45:611-4

8. Lucarelli L. Disturbi dell'alimentazione. In: Ammaniti M, ed. Manuale di psicopatologia dell'infanzia. Milano: Raffaello Cortina Editore; 2001.

9. Ammaniti M, ed. Manuale di psicopatologia dell'infanzia. Milano: Raffaello Cortina Editore; 2001.

10. Lee A, Newman JM. Celiac diet: its impact on quality of life. J Am Diet Assoc 2003;103:1533-5.
11. Nachman F, Planzer del Campo M, González A, et al. Long-term deterioration of quality of life in adult patients with celiac disease is associated with treatment noncompliance. Dig Liver Dis 2010;42:68591.

12. Kolsteren MM, Koopman HM, Schalekamp G, Mearin ML. Health-related quality of life in children with celiac disease. J Pediatr 2001;138:593-5.

13. Kurppa K, Collin P, Mäki M, Kaukinen K. Celiac disease and health-related quality of life. Expert Rev Gastroenterol Hepatol 2011; 5:83-90

14. Chmielewska A, Szajewska H, Shamir R. Celiac disease: prevention strategies through early infant nutrition world. Rev Nutr Diet 2013;108:91-7.

15. Ripamonti C, Clerici CA. Psicologia e salute. Introduzione alla psicologia clinica in ambiente sanitario. Bologna: Il Mulino; 2008.

16. Cimma IG, Furno V, Parziale F, et al. Il progetto vivere la celiachia. Una ricerca sul vissuto emotivo di chi vive in un mondo gluten-free. Available from: http://www.aicpiemonte.it/uploads/media/2 81_file.pdf. Accessed on: September 2013.

17. Quittner AL, Di Girolamo AM, Michel M, Eigen H. Parental response to cystic fibrosis: a contextual analysis of the diagnosis phase. J Pediatr Psychol 1992;17:683-704.

18. Krulik T, Turner-Henson A, Kanematsu Y, et al. Parenting stress and mothers of young children with chronic illness: a cross-cultural study. J Pediatr Nurs 1999;14:130-40.

19. Perricone G, Guerra MP, Cruz 0, et al. Maternal coping strategies in response to a child's chronic and oncological disease: a cross-cultural study in Italy and Portugal. Pediatr Rep 2013;5:e11.

20. Scarzello D. La famiglia del bambino malato cronico. Età Evolutiva 2002;71:106-17.

21. Hanson MJ, Hanline MF. Parenting a child with a disability: a longitudinal study of parental stress and adaptation. J Early Interv 1990;14:234-48.

22. Krauss MW. Child-related and parenting stress: similarities and differences between mothers and fathers of children with disabilities. Am J Ment Retard 1993; 97:393-404.

23. Hadadian, A. Stress and social support in fathers and mothers of young children with and without disabilities. Early Educ Dev 1994;5:226-35.

24. Hoare P, Russell M. The quality of life of children with chronic epilepsy and their families: preliminary findings with a new assessment measure. Dev Med Child Neurol 1995;37:689-96.

25. Abidin, RR. Introduction to the special issue: the stresses of parenting. J Clin Child Psychol 1990;19:298-301.

26. Guarino A, Di Blasio P, D’Alessio M, et al. Parent stress index di Richard Abidin (1990). Firenze: Giunti O.S.; 2008. 